\title{
Pulmonary angiosarcoma presenting with diffuse alveolar hemorrhage: a case report
}

\author{
Min Yu ${ }^{1 \#}$, Weibin Huang ${ }^{1 "}$, Yan Wang ${ }^{2}$, Guangsuo Wang ${ }^{3}$, Lingwei Wang ${ }^{1}$, Weihua Tao ${ }^{1}$, Saadia A. Faiz ${ }^{4}$, \\ Fung Him $\mathrm{Ng}^{5}$, Huiping $\mathrm{Li}^{1 \wedge}$
}

${ }^{1}$ Department of Respiratory and Critical Care Medicine, ${ }^{2}$ Department of Pathology, ${ }^{3}$ Department of Thoracic Surgery, Shenzhen People's Hospital \& the First Affiliated Hospital of Southern University of Science and Technology \& the Second Clinical School of Jinan University \& Shenzhen Municipal Institute of Respiratory Medicine, Shenzhen, China; ${ }^{4}$ Department of Pulmonary Medicine, The University of Texas MD Anderson Cancer Center, Houston, Texas, USA; ${ }^{5}$ Department of Radiology, Princess Margaret Hospital, Hong Kong, China

\#These authors contributed equally to this work.

Correspondence to: Prof. Huiping Li. Department of Respiratory and Critical Care Medicine Shenzhen People's Hospital \& the First Affiliated Hospital of Southern University of Science and Technology \& the Second Clinical School of Jinan University; Shenzhen Municipal Institute of Respiratory Medicine, Shenzhen 518020, China. Email: huipingli2154@hotmail.com.

\begin{abstract}
This study aimed to analyze the diagnosis and treatment of one case of pulmonary angiosarcoma (PPA) retrospectively. The main manifestation of this female patient was cough, hemoptysis and dyspnea. Computed tomography (CT) of the chest revealed multiple small nodules and ground-glass patches in both lungs suggesting of diffuse alveolar hemorrhage (DAH). Laboratory examination revealed decreased hemoglobin and platelet counting, normal coagulation function. Results of rheumatic markers testing including antinuclear antibody (ANA), anti-extractable nuclear antigen antibody (ENA), vasculitis marker, and antiphospholipid antibody were negative. Tumor markers were negative. Sputum smear, sputum culture, and alveolar lavage fluid culture showed negative results. The bone marrow smear was essentially normal. The patient received methylprednisolone pulse therapy $(250 \mathrm{mg}$ daily $\times 5$ days $)$ and immunoglobin $(20 \mathrm{~d}$ daily $\times 7$ days) treatment, but her hemoptysis persisted. Bilateral pleural effusion drainage found a large amount of bloody effusion, but cytology of the pleural fluid showed negative results. The clinical symptoms, laboratory results, imaging findings, and pathological features of the patient were summarized, and problems in diagnosis and treatment were discussed. A thoracoscopic lung biopsy was performed and the diagnosis of PPA was confirmed by pathology and immunohistochemistry (IHC) staining. This case suggested that the possibility of PPA should be considered in patients with DAH, but with negative findings in routine examinations, lung biopsy is usually required.
\end{abstract}

Keywords: Diffuse alveolar hemorrhage (DAH); pulmonary angiosarcoma (PPA); pulmonary biopsy; case report

Submitted Jul 20, 2020. Accepted for publication Dec 29, 2020.

doi: 10.21037/atm-20-7441

View this article at: http://dx.doi.org/10.21037/atm-20-7441

\section{Introduction}

Angiosarcoma is a rare malignancy originating from the vascular endothelium. When tumor cells invade the pulmonary vascular network, they will block and destroy the pulmonary blood vessels, which classically manifests as a pulmonary embolism. Imaging exam may demonstrate the diffuse ground-glass patches and the randomly distributed nodules in both lungs. Most angiosarcoma cases need to be confirmed pathologically by biopsy. Pathological change involves solid nodules consisting of spindle-shaped

^ ORCID: 0000-0002-8691-5740. 
tumor cells, and IHC revealing the presence of at least one positively stained blood vessel-related markers (e.g., CD31 and CD34). Chemotherapy remains the mainstay of treatment for angiosarcoma, although vascular targeted therapy has also been reported. Here, we present a case report of a previously healthy young woman admitted to the hospital with diffuse alveolar hemorrhage (DAH). Comprehensive examinations excluded the typical causes of DAH including infection, vasculitis, and circulatory system diseases. Lung biopsy revealed pulmonary epithelioid hemangioendothelioma. Our experience highlights that in those with DAH of unknown etiology, lung biopsy may facilitate the diagnosis.

We present the following article in accordance with the CARE reporting checklist (available at http://dx.doi. org/10.21037/atm-20-7441).

\section{Case presentation}

All procedures performed in studies involving human participants were in accordance with the ethical standards of the institutional and/or national research committee(s) and with the Helsinki Declaration (as revised in 2013). Written informed consent for publication of the clinical details and/ or clinical images was obtained from the family members of the patient. A 34-year-old woman was admitted with "cough for 5 days and shortness of breath for 3 days". The cough was productive with white sticky phlegm mixed with blood. She visited a local hospital where computed tomography (CT) of the chest revealed multiple small nodules and ground-glass patches in both lungs. Oral ceftazidime for 3 days failed to relieve the symptoms. Her symptoms worsened with dyspnea upon exertion even after walking 100 meters on flat ground. She had no fever. She was admitted to our center. Review of systems was negative, and no change in weight was reported. She also reported a red swollen mass on her left buttock thirty-three days before her admission, and it had gradually enlarged accompanied with bruises and tenderness. She did not receive any treatment for the mass. She worked as a legal officer in a company, and she denied any recent history of contact with live poultry. No significant family history of medical illness was noted. Vital signs were as follows: body temperature $(\mathrm{T}), 36.7^{\circ} \mathrm{C}$; pulse (P), 102 beats/min; respiratory rate (R), 20 breaths/ min; and blood pressure (BP), 112/71 mmHg. Physical exam revealed a 3 to $4 \mathrm{~cm}$ mass with a bruise was seen on her left buttock, and it was slightly firm without fluctuation. On auscultation of the lungs, coarse breath sounds without rales were noted. The heart rhythm was regular, while her abdomen was soft and non-tender. No percussion pain existed in either kidney. There was no peripheral edema.

Auxiliary examinations after admission were also performed. Routine blood test showed the followings: white blood cell (WBC) count, $5.82 \times 10^{9} / \mathrm{L}$; hemoglobin concentration, $53 \mathrm{~g} / \mathrm{L}$; and platelet count, $83 \times 10^{9} / \mathrm{L}$. Routine urine test showed the followings: red blood cell (RBC) count, 75 cells $/ \mu \mathrm{L}$; WBC count, $25 / \mu \mathrm{L}$; and protein, negative. Liver function test showed the followings: lactate dehydrogenase, $274 \mathrm{U} / \mathrm{L} ; \alpha$-hydroxybutyrate dehydrogenase, $212 \mathrm{U} / \mathrm{L}$; total protein, $53.6 \mathrm{~g} / \mathrm{L}$; serum albumin, $35.3 \mathrm{~g} / \mathrm{L}$; direct bilirubin, $7.21 \mu \mathrm{mol} / \mathrm{L}$; and immunoglobulins, $18 \mathrm{~g} / \mathrm{L}$. Renal function was normal: complement C4, $0.08 \mathrm{~g} / \mathrm{L}$; immunoglobulin A, $0.49 \mathrm{~g} / \mathrm{L}$; immunoglobulin $\mathrm{G}, 6.46 \mathrm{~g} / \mathrm{L}$; and total iron binding capacity, $46 \mu \mathrm{mol} / \mathrm{L}$. Measurement of inflammation markers showed the following: hypersensitive $\mathrm{C}$-reactive protein, $2.21 \mathrm{mg} / \mathrm{L}$; RBC sedimentation rate, $14 \mathrm{~mm} / \mathrm{h}$; and serum procalcitonin (PCT), negative. Testing for coagulation function showed the following: prothrombin time, 14.3 seconds; activated partial thromboplastin time, 29.2 seconds; and fibrinogen, $1.75 \mathrm{~g} / \mathrm{L}$. Thyroid function test showed that the total triiodothyronine $\left(\mathrm{TT}_{3}\right)$ level was $0.95 \mathrm{nmol} / \mathrm{L}$. Testing for rheumatic markers including ANA, ENA antibody, vasculitis marker, and antiphospholipid antibody showed negative results. Tumor markers were negative. Sputum smear, sputum culture, and alveolar lavage fluid culture showed negative results. The bone marrow smear was essentially normal. It revealed that bone marrow growth was roughly normal (about 60\%), the myeloid/ erythroid ratio decreased, myeloids and erythroids were present at different stages (dominantly myelocytes or polychromatophilic erythroblasts), megakaryocytes (mainly multilobated nuclear megakaryocytes) were visible, and no abnormal tumor cells were present. Plain lung scan plus computed tomography pulmonary angiogram (CTPA) revealed no clear pulmonary artery filling defects on CTPA, but did find diffuse ground-glass patches in both lungs (Figure 1). The finding from cardiac color Doppler ultrasound was roughly normal. Abdominal color Doppler ultrasound and urinary tract ultrasound showed no obvious abnormality. Ultrasound of the mass in the left buttock showed a mixed subcutaneous lesion in left buttock, and no obvious blood flow signal was observed in it.

After the patient was admitted, she was supported with a respiratory humidification treatment device and received antimicrobial therapies including imipenem, voriconazole, 

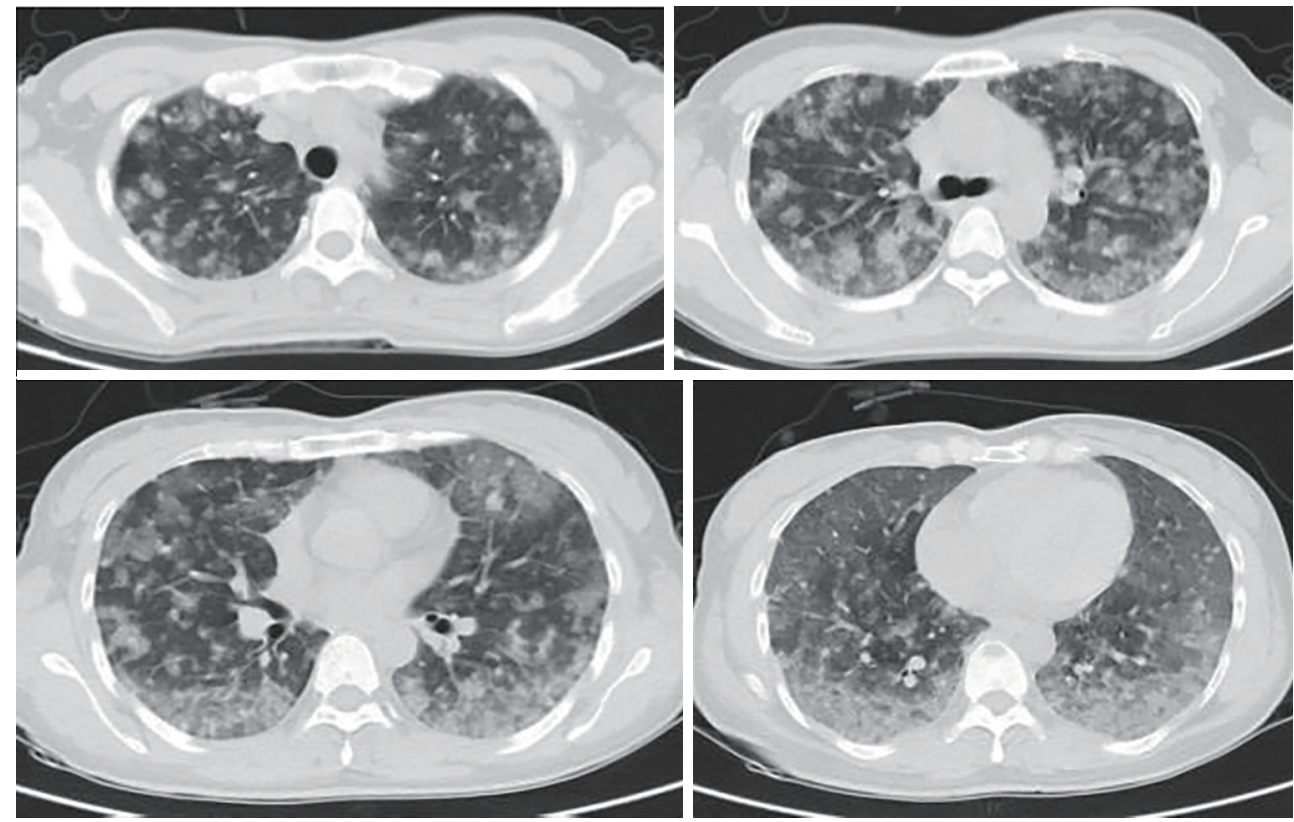

Figure 1 Chest CT on the day of admission showing multiple small nodules and ground-glass patches in both lungs. CT, computed Tomography.

and moxifloxacin. However, her hemoptysis persisted. The daily volume of hemoptysis was about $200 \mathrm{~mL}$, and the patient was repeatedly infused with hemoglobin and plasma. Her blood oxygen saturation gradually decreased, and the patient was intubated 4 days after admission and started mechanical ventilation. Methylprednisolone pulse therapy ( $250 \mathrm{mg}$ daily $\times 5$ days) was initiated on day 7; however, the hemoptysis persisted. On day10, bilateral pleural effusion was found on a bedside chest radiograph. Thoracentesis was then performed and a large amount of bloody pleural effusion was drained. However, cytology in the pleural fluid showed negative results. On day 16, a second chest CT (Figure 2) showed significant increase in multiple nodules and diffuse infiltrates throughout both lungs. The possibility of pulmonary angiosarcoma (PPA) was considered. After consulting with her family members and completing the preoperative preparations, a thoracoscopic lung biopsy was performed on day 16 . The pathological findings are shown in Figure 3. The lower left lung tissue was sampled and paraffin-fixed for preparing sections. Hemorrhage and a large amount of fibrinous exudation and inflammatory exudation were observed in the lung tissue. Infiltration of a large number of foam-like tissue cells was seen in the alveolar cavity. Pleomorphic cells were seen locally. Combined with IHC findings, these results suggested a diagnosis of epithelioid angiosarcoma. The IHC findings were as follows: CD34, +; CD31, +; HMB45, -; melan-A, -; napsin A, -; P40, -; TTF1, -; EMA, -; ERG, +; FLI-1, +; S100, -; CK, -; and Ki67, 70\%+. The patient had a hypovolemic shock after surgery and was transferred to the ICU for resuscitation. She was given intermittent blood transfusion and vasoactive agents to maintain hymodynamic stablility, but her hemoptysis persisted. She died of respiratory failure on day 19.

\section{International multidisciplinary team (iMDT) discussion}

\section{Discussion among physicians from Shenzhen People's Hospital}

This case report is aimed to initiate a multidisciplinary consultation on the diagnosis and treatment of PPA, presenting with DAH. The clinical symptoms, laboratory results, imaging findings, and pathological features of the patient were summarized, and problems in diagnosis and treatment were discussed. In patients with DAH, PPA should be kept in the differential, and in those without clear etiology, lung biopsy is usually required. 

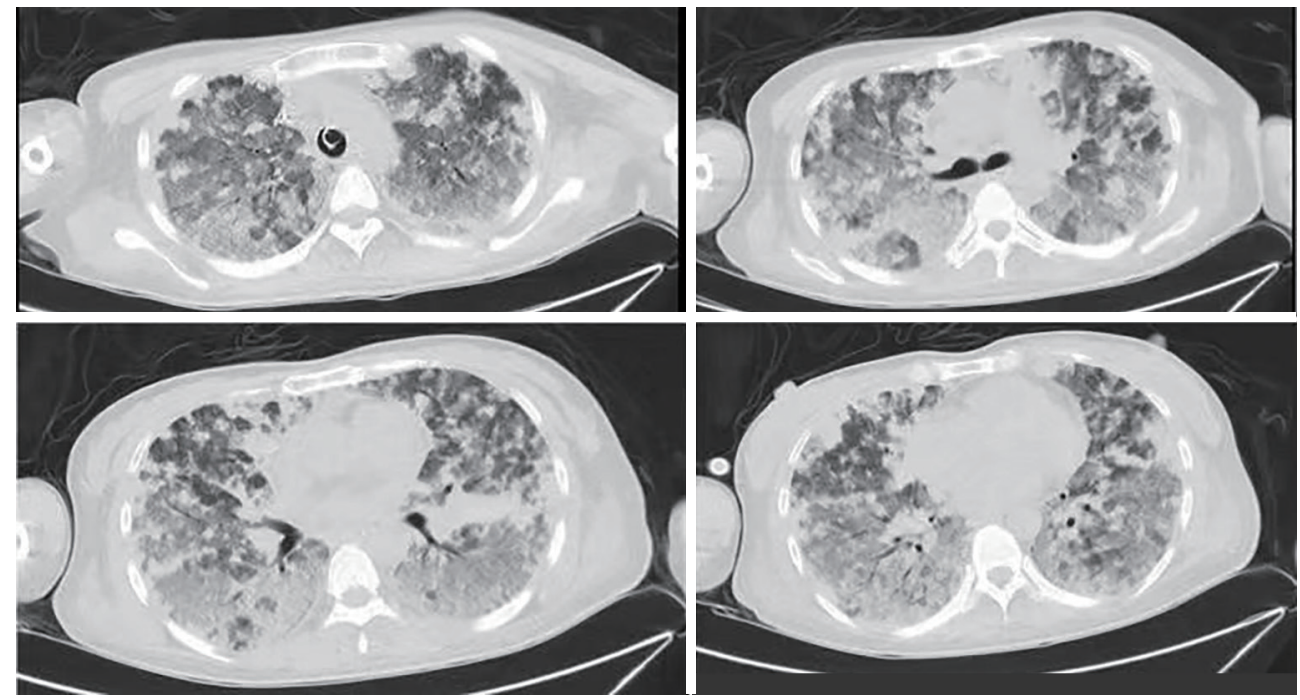

Figure 2 A second chest CT performed two weeks after admission showing the presence of multiple nodules and significantly increased exudation in both lungs. CT, computed tomography.

\section{Department of Respiratory Medicine}

The patient had DAH, with unknown etiology. DAH is a clinical syndrome defined by a disturbance of the alveolarcapillary basement membrane that causes bleeding into the pulmonary alveoli. Its main manifestations include hemoptysis, anemia, diffuse lung exudation, and hypoxic respiratory failure. Histopathologically, it can have the following three features: pulmonary capillaritis, bland pulmonary hemorrhage, and diffuse alveolar damage. Pulmonary capillaritis is caused by the infiltration of neutrophils into the alveolar septum, which subsequently leads to necrosis of the alveolar septum and incomplete capillary structure. Bland pulmonary hemorrhage refers to hemorrhage occurring in the alveolar cavity, but with no inflammation or destruction of the alveolar structure; it may result from hemorrhagic disease or be a complication of anticoagulation therapy. DAH is accompanied with edema of the alveolar septal wall and formation of hyaline membrane in the alveolar cavity. Common causes of DAH include systemic vasculitis, anti-glomerular basement membrane disease, rheumatic connective tissue disease, infection, use of drugs, and circulatory system diseases. In rare cases it can also be caused by tumors (1). Systemic vasculitis that can cause DAH includes antineutrophil cytoplasmic antibody (ANCA)-related vasculitis, microscopic polyangiitis, and Behcet's disease. Rheumatic connective tissue diseases such as systemic lupus erythematosus and mixed connective tissue disease can also cause similar symptoms. However, most of these diseases have increased inflammation markers or positive rheumatoid factors (2). Infection is another common cause of DAH. Depending on the host's immune status, the pathogen also differs. The main pathogens causing DAH in immunocompromised patients include cytomegalovirus, adenovirus, Aspergillus, Mycoplasma, Legionella, and Strongyloides. For patients with normal immune function, the typical infectious diseases that lead to DAH include H1N1 influenza, dengue fever, leptospirosis, malaria, and Staphylococcus aureus infection (3). However, since the immune/inflammation markers were not elevated and the signs of infection were not obvious in this patient, infection or vasculitis might not have been the cause of the patient's DAH.

\section{Department of Rheumatology and Immunology}

The patient had no increase in inflammatory indicators, and her rheumatic factors were also negative. After highdose methylprednisolone pulse treatment for 5 days, the patient's hemoptysis, hypoxia and infiltrates showed no improvement. Thus, a diagnosis of vasculitis or rheumatic connective tissue diseases could not be supported. Further examinations were needed to rule out the possibility of a malignancy or hematological diseases. 

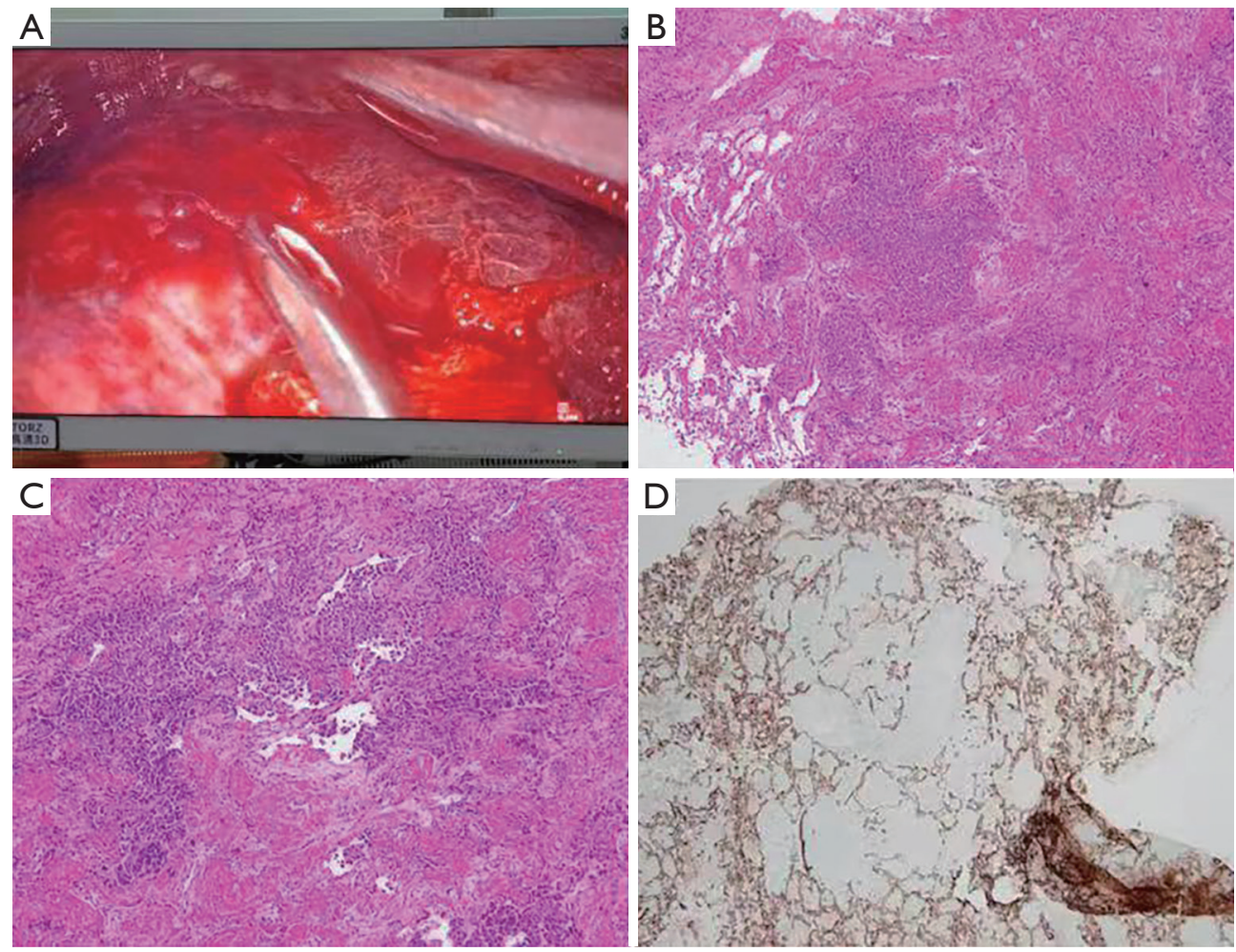

Figure 3 Images of thoracoscopic lung biopsy. (A) Diffuse hemorrhage on the surface of lung tissue, along with scattered nodular lesions, was seen under intraoperative thoracoscopy; (B) HE staining of pathological section showing the tumor nodules and the necrotic tissues around the nodules (100x); (C) high-power microscopy of HE staining showing (200x) a tumor composed of epithelioid histiocytes, with more frequent mitotic phase; (D) immunohistochemical staining showing tumor cells stained positively for CD34 which was a marker of vascular endothelial cells, suggesting they were vascular-derived cells (100x). HE, hematoxylin eosin.

\section{Department of Infectious Diseases}

The patient had no fever and her renal function was normal. Urine RBC count was slightly elevated. Chest CT revealed diffuse lesions. Repeated examinations of sputum smears/ cultures, bronchoalveolar lavage (BAL) fluid and culture, and blood high-throughput sequencing showed negative results. Empiric antibacterial treatment with meropenem and voriconazole did not improve the symptoms. Since there was no adequate evidence to support a diagnosis of infection and the immune/inflammatory markers were not high, a hematological disease or a malignancy was more likely to be the cause.

\section{Department of Hematology}

The patient had normal coagulation function. Peripheral blood smear test, bone marrow smear test, and bone marrow biopsy showed normal results. There was no abnormality on superficial lymph node ultrasound, and there was no evidence of circulatory system disease. Chest, abdomen and pelvic CT showed no evidence of solid tumors. Further examinations and tests would be required to gather more relevant evidence. Lung biopsy should be taken into consideration if necessary.

\section{Department of Pathology}

After repeated discussions among the multidisciplinary team (MDT), the possibility of a pulmonary vascular tumor was considered. After communicating with the family members of the patient, a thoracoscopic lung biopsy was performed. A diagnosis of PPA was confirmed by pathology and IHC staining. 
Several issues regarding to the diagnosis and treatment of this patient were further discussed as follows

Question 1: What are the potential emergency treatments for patients with DAH before a definite diagnosis is made?

\section{Expert opinion1: Dr. Fung Him Ng, Department of} Radiology, Princess Margaret Hospital, Hong Kong, China

If there is massive haemoptyosis, a part of medical treatment using transamin and correct the clotting profile by transfusion of platelet and fresh frozen plasma (FFP), the bleeding could be stopped by bronchoscopy or minimal invasive methods, e.g., bronchial artery embolization. CT bronchial arteriogram should be performed before the embolization to help to delineate the vascular anatomy and localize the exact site of haemoptysis. Embolization coils, glue or gelofoam could be used to block the bleeding vessels of the tumor causing the haemophysis.

Expert opinion2: Dr. Saadia A. Faiz, Department of Pulmonary Medicine, The University of Texas MD Anderson Cancer Center, Houston, USA

DAH is a life-threatening condition, and the focus of emergent treatment includes management of hypoxemia and therapies directed towards the underlying cause. Ventilatory support with supplemental oxygen is initiated and often escalated to high-flow oxygen, non-invasive or mechanical ventilation. Systematic evaluation for the disease process including work up for vasculitis or autoimmune process, evaluation of pharmacologic or illicit drug use, aberrant hematologic parameters, and infectious pathogens. Initiation of systemic glucocorticoids is often the first response to treat a potential steroid-responsive disease or to quell the inflammatory response. Once the disease is confirmed, additional immunosuppressive medications (including cyclophosphamide or rituximab) or plasmapheresis may be considered.

\section{Question 2: How can the proper indication and timing of lung biopsy be determined?}

\section{Expert opinion1: Dr. Fung Him Ng}

Early diagnosis of primary PPA is uncommon because of the non-specific respiratory manifestations and consequent low index of suspicion. Definitive diagnosis is made on the basis of histopathological and immunohistochemical findings. Unresolved pulmonary lesion or lesion progression should arrange earliest possible biopsy to exclude malignancy.

\section{Expert opinion2: Dr. Saadia A. Faiz}

If clinical history or diagnostic workup are unrevealing for an etiology, surgical lung biopsy may be required to establish the diagnosis as in the case described (1).

\section{Question 3: What are the currently available effective treatment options for PPA?}

\section{Expert opinion1: Dr. Fung Him Ng}

No standard treatment was recommended specifically for pulmonary epithelioid angiosarcoma. Surgery, radiation therapy, and chemotherapy have all been attempted, among which surgery was the mainstay for locally confined disease (2). Previous studies have also shown that angiosarcoma was radiosensitive (3). Clinical trials demonstrated that chemotherapeutic combinations such as doxorubicin/ifosfamide and docetaxel/gemcitabine were partial and full effective (4). Systemic administration of high doses of recombinant interleukin 2 (rIL-2) also seems to have been effective (5). Although many attempts have been made to treat primary PPA, these tumors have a very aggressive clinical course and a grim prognosis.

\section{Expert opinion2: Dr. Saadia A. Faiz}

PPA is a rare entity, and in those affecting the pulmonary artery, surgical resection with neoadjuvant chemotherapy is the therapy of choice (6). However, presentation with DAH is even more sparse in the medical literature, thus treatment options have not been well studied. Therapeutic modalities including radiation, various chemotherapies, and surgical resection have all been described, but none have been demonstrated to be significantly effective $(7,8)$.

\section{Conclusions}

Angiosarcoma is a rare malignancy originating from the vascular endothelium, accounting for $1-2 \%$ of soft tissue sarcomas. The average age of onset is 45 years, and there is no obvious gender predominance. It can originate from multiple tissues/organs such as the skin, liver, and heart. PPA can be either primary or metastatic. Primary PPA is relatively rare, and its CT findings (e.g., intravascular filling defects of contrast agent and narrowing of the luminal space) are similar to those of pulmonary embolism (9). Metastatic lesions from other sites (mainly heart and liver) are more common, accounting for about $60-80 \%$ 
of all PPA cases $(10,11)$. When the tumor cells invade the pulmonary vascular network, they will block and destroy the pulmonary blood vessels, which clinically manifests as DAH and pulmonary embolism, with the main symptoms including chest pain, cough, and intermittent fever. In this case, anti-coagulatory treatment should be avoided since it would induce severe hemorrhage. The imaging findings may include diffuse ground-glass shadows in both lungs and randomly distributed nodules. Clinically, it is often misdiagnosed as vasculitis and infectious disease. Although genetic researches have tried to clarify the gene expression profiling of angiosarcoma, there was no biomarker available for early diagnosis so far. Most angiosarcoma cases need to be confirmed pathologically by biopsy. Pathology is confirmed when solid nodules consisting of spindle-shaped tumor cells are apparent and when hemorrhage can be seen in the tumor nodules and adjacent lung parenchyma. IHC may reveal the presence of at least one positively stained blood vessel-related marker (e.g., CD31 and CD34) (12). Chemotherapy including anthracycline and taxanes remains the mainstay of treatment for a subset of patients with limited durability. Vascular targeted therapy with tyrosine kinase inhibitors such as pazopanib, sorafenib and regorafenib have also been reported but warrants further validation (13). The role of surgery in treating primary PPA has been explored in the literature (14).

In our case, there were no obvious filling defects on CTPA, and chest CT showed diffuse ground-glass shadows in both lungs. A mass was found on her left buttock. The possibility of the metastasis of the hemangioendothelioma from the left hip muscle layer to both lungs was high. Unfortunately, although the mass in the left buttock was noted at admission, no further biopsy was performed, or early diagnosis of angiosarcoma might be obtained if the PPA was metastatic. The patient only had repeated ultrasound scans and aspiration all of which yielded dark bloody fluids. Therefore, it was impossible to identify whether the PPA was primary or metastatic.

\section{Acknowledgments}

Funding: None.

\section{Footnote}

Reporting Checklist: The authors have completed the CARE reporting checklist. Available at http://dx.doi.org/10.21037/ atm-20-7441
Conflicts of Interest: All authors have completed the ICMJE uniform disclosure form (available at http://dx.doi. org/10.21037/atm-20-7441). The authors have no conflicts of interest to declare.

Ethical Statement: The authors are accountable for all aspects of the work in ensuring that questions related to the accuracy or integrity of any part of the work are appropriately investigated and resolved. All procedures performed in studies involving human participants were in accordance with the ethical standards of the institutional and/or national research committee(s) and with the Helsinki Declaration (as revised in 2013). Written informed consent for publication of the clinical details and/or clinical images was obtained from the family members of the patient.

Open Access Statement: This is an Open Access article distributed in accordance with the Creative Commons Attribution-NonCommercial-NoDerivs 4.0 International License (CC BY-NC-ND 4.0), which permits the noncommercial replication and distribution of the article with the strict proviso that no changes or edits are made and the original work is properly cited (including links to both the formal publication through the relevant DOI and the license). See: https://creativecommons.org/licenses/by-nc-nd/4.0/.

\section{References}

1. Lara AR, Schwarz MI. Diffuse alveolar hemorrhage. Chest 2010;137:1164-71.

2. Kojima K, Okamoto I, Ushijima S, et al. Successful treatment of primary pulmonary angiosarcoma. Chest 2003;124:2397-400.

3. Sasaki R, Soejima T, Kishi K, et al. Angiosarcoma treated with radiotherapy: impact of tumor type and size on outcome. Int J Radiat Oncol Biol Phys 2002;52:1032-40.

4. Wilson R, Glaros S, Brown RK, et al. Complete radiographic response of primary pulmonary angiosarcomas following gemcitabine and taxotere. Lung Cancer 2008;61:131-6.

5. Palvio DH, Paulsen SM, Henneberg EW. Primary angiosarcoma of the lung presenting as intractable hemoptysis. Thorac Cardiovasc Surg 1987;35:105-7.

6. Blackmon SH, Rice DC, Correa AM, et al. Management of primary pulmonary artery sarcomas. Ann Thorac Surg 2009;87:977-84.

7. Patel AM, Ryu JH. Angiosarcoma in the lung. Chest 1993;103:1531-5. 


\section{Page 8 of 8}

8. Shirey L, Coombs D, Talwar A, et al. Pulmonary epithelioid angiosarcoma responsive to chemotherapy: A case report. Radiol Case Rep 2018;13:479-84.

9. Ma J, Zhang W, Huang Y, et al. Radiologic findings of primary pulmonary angiosarcoma: A case report. Medicine (Baltimore) 2018;97:e11105.

10. von Ranke FM, Zanetti G, Hochhegger B, et al. Infectious diseases causing diffuse alveolar hemorrhage in immunocompetent patients: a state-of-the-art review. Lung 2013;191:9-18.

11. Wang H, Shi J, Liu H, et al. Clinical and diagnostic features of angiosarcoma with pulmonary metastases: A

Cite this article as: Yu M, Huang W, Wang Y, Wang G, Wang L, Tao W, Faiz SA, Ng FH, Li H. Pulmonary angiosarcoma presenting with diffuse alveolar hemorrhage: a case report. Ann Transl Med 2021;9(1):74. doi: 10.21037/atm$20-7441$
Yu et al. Angiosarcoma manifests as diffuse alveolar hemorrhage

retrospective observational study. Medicine (Baltimore) 2017;96:e8033.

12. Ren Y, Zhu M, Liu Y, et al. Primary pulmonary angiosarcoma: Three case reports and literature review. Thorac Cancer 2016;7:607-13.

13. Tanimoto A, Takeuchi S, Kotani H, et al. Pulmonary carcinosarcoma showing an obvious response to pazopanib: a case report. BMC Pulm Med 2018;18:193.

14. Yin K, Zhang Z, Luo R, et al. Clinical features and surgical outcomes of pulmonary artery sarcoma. J Thorac Cardiovasc Surg 2018;155:1109-15.e1. 